\title{
Relativistic Shock Waves and the Excitation of Plerions
}

\author{
Jonathan Arons ${ }^{1,2}$, Yves A. Gallant ${ }^{2}$, Masahiro \\ Hoshino, A. Bruce Langdon ${ }^{3}$ and Claire E. Max \\ Institute of Geophysics and Planetary Physics, \\ Lawrence Livermore National Laboratory
}

To be published in the Proceedings of IAU Colloquium No. 128, 'The Magnetospheric Structure and Emission Mechanisms of Radio Pulsars'

1. Department of Astronomy, University of California at Berkeley

2. Department of Physics, University of California at Berkeley

3. X-Division, Lawrence Livernore National Laboratory 


\begin{abstract}
The shock termination of a relativistic magnetohydrodynamic $w$. d from a pulsar is the most interesting and viable model for the excitation of the synchrotron sources observed in plerionic supernova remnants. We have studied the structure of relativistic magnetosonic shock waves in plasmas composed purely of electrons and positrons, as well as those whose composition includes heavy ions as a minority constituent by number. We find that relativistic shocks in symmetric pair plasmas create fully thermalized distributions of particles and fields downstream. Therefore, such shocks are not good candidates for the mechanism which converts rotational energy lost from a pulsar into the nonthermal synchrotron emission observed in plerions. However, when the upstream wind contains heavy ions which are a minority constituent by number density, but carry the bulk of the energy density, much of the energy of the shock goes into a downstream, nonthermal power law distribution of positrons with energy distribution $N(E) d E \propto E^{-s}$. In a specific model presented in some detail, $s=3$. These characteristics are close to those assumed for the pairs in macroscopic MHD wind models of plerion excitation. The essential mechanism is collective synchrotron emission of left-handed extraordinary modes by the ions in the shock front at high harmonics of the ion cyclotron frequency, with the downstream positrons preferentially absorbing almost all of this radiation, mostly at their fundamental (relativistic) cyclotron frequencies. Possible applications to models of plerions and to constraints on theories of energy loss from pulsars are briefly outlined.
\end{abstract}




\section{Background}

The excitation of diffuse, nonthermal astrophysical synchrotron sources by energy lost from central compact objects has long been a puzzle in high energy astrophysics. Pulsars and their surrounding plerionic nebulae form the nearest at hand and best studied examples of this problem. Other examples include the excitation of extragalactic radio sources by jets, and possibly the emission from active galactic nuclei themselves. The Crab Nebula is the best studied of the plerions, and for our purpose here, we will take the physical problems it poses to be typical of the class.

The X-ray and gamma-ray emission from the Crab requires continuous energy input, which is aptly explained by the energy losses from a central, rotating magnetized neutron star (Pacini 1967). Indeed, the identification of this idea with the subsequently discovered pulsars (Gold 1968 ) is one of the pillars of the reasoning by which we identify the rotating neutron star model with the observed pulsar phenomenon. However, the physics of the energetic link has remained an unsolved problem. The Crab has features similar to a wide variety of astrophysical synchrotron sources (power from a central compact object, a large scale magnetic field, diffuse, optically thin nonthermal synchrotron emission, dense filaments of thermal emission line plasma embedded in the diffuse nonthermally emitting gas). Therefore, an understanding of the physical mechanisms through which energy is carried, without emitting many photons, from the central compact object to the surrounding environment, then degraded into the observed nonthermal emission, has wide astrophysical significance. In addition, the nature of this conversion mechanism may provide constraints on models of the pulsar magnetosphere.

Relativistic, magnetohydrodynamic (MHD) models of the coupling between pulsar and nebula are the most successful at the present time. Building on earlier ideas (Piddington 1957, Rees and Gunn 1974, Kundt and Krotscheck 1980), Kennel and Coroniti (1984a,b) showed that such a wind from the pulsar, terminated by a standing, transverse magnetosonic shock wave, could give a good account of the nebular dynamics and of the nevular spectrum of high energy photons (near infrared, optical, X-rays and gamma rays), which are an instantaneous probe of the pulsar's outflow. The prominent new results of their model are several. 1) The post shock flow fits into the slowly expanding nebula only if the upstream flow is almost entirely dominated by kinetic energy, with

$$
\sigma \equiv \frac{B_{1}^{2}}{4 \pi m N c^{2} \gamma_{1}} \simeq 0.003
$$

Subsequent work by Emmering and Chevalier (1987), who included the time dependence introduced by the nonrelativistic nebular expansion velocity, confirm this result, with an increase of $\sigma$ to perhaps 0.005 . The dynamics of the model provide a natural explanation of the apparent equipartition of the fields with the relativistic plasma in the main body of the nebula, since the decelerating flow behind the shock compresses the field until approximate equipartition is reached and no further deceleration is possible. 2) If the upstream flow is entirely composed of pairs (an assumption), and if the post-shock energy distributions are assumed to power laws with $N(E) \propto E^{-s}$ (another assumption), then the synchrotron emission from the downstream flow can be fit to the observed X-and gamma ray spectra of the Nebula, taken as an unresolved 
object, if $s \approx 2.3$. Once this fit is made, the optical and near infrared spectra of the nebula (again taken as an unresolved object) are predicted (more or less) correctly by the model.

This theory is sufficiently successful as a macroscopic explanation of the phenomenology to provide a setting for investigation of the microscopic physics. Our studies began from several questions. Does a relativistic, magnetosonic shock in a pair plasma actually create the assumed power law distributions downstream? If so, what is the mechanism of acceleration in such shocks? Are there other observable consequences of this mechanism, other than the desired particle acceleration, which allow one to test the shock hypothesis directly? We emphasize that one cannot appeal to the ever popular ideas of diffuse shock acceleration proposed for particle acceleration by interplaneiary and interstellar shocks (e.g., Bell 1978, Ellison et al 1990), since the termination shocks of pulsar winds must be fairly close to having the magnetic field perpendicular to the flow upstream - indeed, for the Crab, a larninar Archimedean spiral in the outflow from the pulsar would have $\Theta_{B n}$, the angle between the flow direction and the magnetic field, differing from $90^{\circ}$ only by one part in $10^{9}$ ! In addition, in relativistic shocks, the particles can stream ahead of the shock and participate in the back and forth motion across the front only when $\Theta_{B n}<1 / \gamma_{1}$ (Begelman and Kirk 1990), where $\gamma_{1}$ is the Lorentz factor of the upstream flow. Therefore, some other mechanism of shock acceleration must apply. In relativistic shocks, the mechanisms of high energy particle acceleration and of basic thermalization can be one and the same - accelerating relativistic particles can be part of the problem of shock structure itself.

\section{Shock Structure and Particle Acceleration}

Pulsar outfiows must have extremely high Mach number. The MHD model for the Nebula works if the upstream 4-speed in the shock frame is $u_{1} \cong \gamma_{1} \geq 10^{6}$, a number which is consistent with some (but by no means all) suggestions for the outflow from the magnetosphere. The Alfven Mach number in the flow is $M_{A}=u_{1} / \sqrt{\sigma} \cong 1.5 \times 10^{7}$ ! Under these circumstances, magnetic reflection of particles from the shock front plays an essential role in the shock dynamics. Solitary waves with unidirectional flow exist only for $M_{A}<1+1 / \gamma$ (Kennel and Pellat 1976, Chiueh 1989). At higher Mach number, incoming particles reflect from the enhanced magnetic field in the wave front and are set into Larmor gyration, leading to a new kind of magnetosonic soliton with reflected particles self-consistently incorporated (Alsop and Arons 1988). This reflection process requires all the momentum of the incoming plasma to be temporarily stored in a magnetic overshoot, with $B_{\text {peak }} / B_{1}=\left(1+\sigma^{-1}\right)^{1 / 2}$. The dependence of the overshoot on $\sigma$ alone is characteristic of relativistic magnetosonic shocks - once $\gamma_{1} \gg 1$, the dependence on $\gamma_{1}$ itself is entirely subsumed in the scale of the flow being that of the Larmor radius based on the upstream parameters. $\sigma$ provides a better parameterization than does $M_{A}$.

While it is possible to construct solitary wave models with magnetically reflected particles in pair plasmas (Alsop and Arons 1988), unraveling the instabilities and thermalization mechanisms in this highiy inhomogeneous environment is a formidable task. We turned to fuly non-linear, fully sef-consistent particle-in-cell numerical simulations in order to find out what is happening. The basic method is to integrate the equations of motion of many charged particles in their selfconsistent fields. Suppose at some time $t_{n}$, one knows the electromagnetic field on a spatial grid, anci one knows the positions and momenta of all the particles $\left\{\mathbf{x}_{j}\left(t_{n}\right), \mathbf{p}_{j}\left(t_{n}\right)\right\}$ (they are not on 
the grid). One then finds the Lorentz force at each particle's position and uses Newton's law (in relativistic form) to advance the particles to new phase space positions $\left\{\mathbf{x}_{j}\left(t_{n}+d t\right), \mathbf{p}_{j}\left(t_{n}+d t\right)\right\}$. One then calculates new fields at time $t_{n}+(d t / 2)$ using the charge densities and currents at time $t_{n}$, with the particles distributed on the grid with a suitable weight function. These new fields are used to advance the positions of the particles once again, with the time step set by by various stability considerations. These techniques are quite well understood and have been in use for 20 years (Langdon and Lasinski 1976, Birdsall and Langdon 1985).

We modeled shock waves in pair plasmas by setting up a one-dimensional spatial grid in the computer, with length anywhere from 10 to 40 Larmor radii based on the magnetic field and particle energy in the upstream flow. The flow and field geometry is shown in Figure 1.

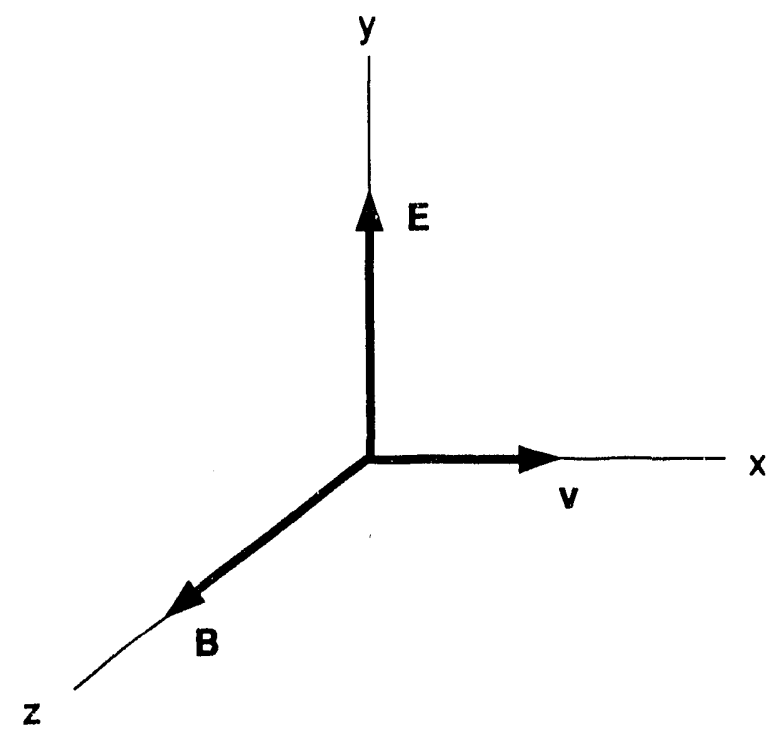

Figure 1: Upstream geometry of flow, magnetic field and electric field. The electrostatic field parallel to $\mathrm{v}$ is zero upstream, while the electric field has magnitude $E=(v / c) B$.
At the initial time, the cold plasma filis the computational box, with flow Lorentz factor $\gamma_{1} \gg 1$ and a magnetic field polarized across the flow carried with the plasma at the fluid speed. The ratio of magnetic energy density to flow energy density upstream $\sigma$ is the only parameter of significance. As expected from theoretical considerations, simulations with the same value of $\sigma$ but differing $\gamma_{1}$ yield identical results, when $\gamma_{1} \gg 1$. We have surveyed parameter space with these spatially $1 \mathrm{D}$ models from $\sigma=13$ down to $\sigma=0.001$.

At the injection point (the "left wall"), the particles are injected all with the same upstream velocity, and the magnetic is carried with the fluid, by requiring the boundary electric field to be that of a perfectly conducting fluid. The opposite end of the grid is represented as a conducting rubber wall -

particles bounce off the wall, which acts as a perfect conductor as far as the fields are concerned. It would be better to have outflow boundary conditions on the plasma and radiation, but so far a useful algorithm to implement these in the dense downstream plasma has not been constructed. Because the magnetic field points across the flow, the wall has no influence on the shock structure, once the point of particle reflection is more than a few Larmor radii away from the wall. In the low $\sigma$ shocks of interest here, the wave energy is never more than a few percent of the thermal and kinetic energy of the plasma, so the conducting wall, which forces the downstream waves to be standing modes, has little influence on the shock once it its well upstream of the wall.

The bouncing of the particles off the wall initiates the shock wave. After a confused initial adjustment, a well developed shock appears and travel upstream. Figure 2 shows the electromagnetic field structure of a shock located at $x=21$ in a pure pair plasma, with $\gamma_{1}=10^{6}$ 
and $\sigma=0.01$, as observed 34 cyclotron periods after the plasma bounced off the simulation wall. One finds an intense, quasi-coherent electromagnetic precursor travelling in front of the shock, while downstream, a few per cent of the total energy is turned into a linearly polarized Rayleigh-Jeans spectrum of electromagnetic waves. Figure 2 also shows the phase space of the electrons in the whole box at a fixed time in the flow. One sees the reflection of the particles from the shock front, followed by rapid thermalization of the Larmor gyration. The downstream spectra of electrons and positrons is essentially identical to the Maxwellian electron distribution shown in Figure 5 below. The Rayleigh-Jeans distribution (not shown) of down stream waves has a temperature close to the post-shock temperature of the plasma. Thus, transverse shocks in a pure pair plasma create a fully thermalized downstream medium, contrary to a basic assumption of the MHD model of plerionic supernova remnants.
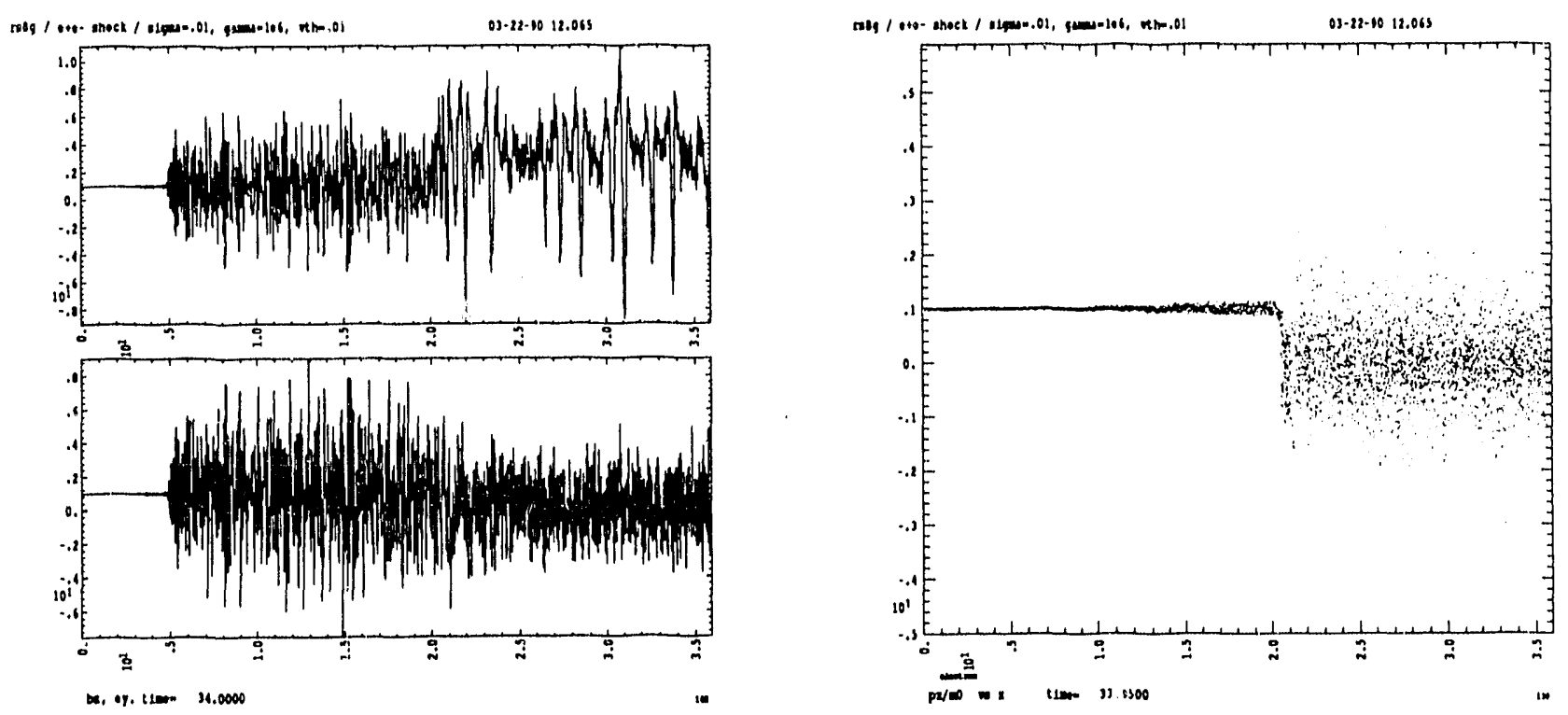

Figure 2: Structure of a relativistic shock in a symmetric pair plasma with $\sigma=0.01$ and $\gamma_{1}=10^{6}$. Upper left panel: magnetic field. The shock occurs at $x=21$, where the unit of length is the Larmor radius of the electrons and positrons based on the upstream flow parameters. An intense electromagnetic precursor propagates ahead of the shock. Lower left panel: transverse electric field, showing the strong precursor and the weaker downstream extraordinary mode fluctuations. Upstream, the mean value of the electric field is $E_{1}=\left(v_{1} / c\right) B_{1} \approx B_{1}$, while downstream, the mean value of the electric field is zero, since the simulation is done in the frame where the downstream plasma is at rest. Right panel: The $p_{x}-x$ projection of the electrons' phase space, showing the cold plasma injected at $x=0$. The large amplitude precursor increases the momentum "dispersion" upstream of the shock. The mechanical shock transition occurs where the particles are magnetically reflected from the shock front, at $x=21$, with almost immediate thermalization of the plasma. 
Some initial results of these calculations were reported by Langdonet al (1988), and full details are given by Gallant et al (1990). We have done a few preliminary calculations with the magnetic field oblique to the flow, using what is otherwise the same $1 \mathrm{D}$ model. So long as $\Theta_{B n}>50^{\circ}$, the results are semiquantitatively the same as for the purely transverse case, as one might suspect since the structure is dominated by the refelcted particles and the rapid dissipation. Smaller obliquities may be different, but for technical reasons this conclusion is not yet warranted. We have also investigated some aspects of the two dimensional structure of these shocks, motivated by the observation that the 1D model leaves the plasma with a high temperature in the momenta orthogonal to the magnetic field, while it is still cold in the momenta along $B$. Such a medium is unstable, most prominently (at low $\sigma$ ) with respect to the Weibel instability. In one $2 \mathrm{D}$ shock calculation, we have found this effect does heat the plasma in the direction along $\mathrm{B}$, as well as broaden the distribution of gyrational momenta, but the basic shock structure persists, with downstream anisotropy $T_{\perp} / T_{\|} \sim 2$. As this kind of anisotropy may be observationally relevant (Michel et al 1990) to the pattern of optical polarization in the Crab Nebula, further 2D work is of interest, for a different version of the shock model which shows a more interesting downstream particle spectrum.

The nature of the thermalization mechanism gives a clue to a shock model which does have nonthermal particle acceleration. As seen in the shock frame, the reflected particles form a gyrating ring with small momentum dispersion in the leading edge of the shock. Such rings are unstable - they form synchrotron masers (Zheleznyakov and Suvorov 1972, Yoon 1990, Hoshino and Arons 1990, Arons et al 1990). Calculations of the growth rate for a homogeneous pair plasma, along with estimates of the maximum harmonic number that can be generated, suggest that the dissipation observed in the pure pair plasmas is due to the formation of synchrotron masers, with consequent plasma heating as the extraordinary modes radiated are absorbed.

Now imagine what happens if the upstream flow contains heavy ions, as well as pairs. Since all the species flow into the shock with the same speed (the upstream Larmor radii are small compared to the overall flow scale in which the shock is embedded), $Z N_{1 i}+N_{1+}=N_{1-}$. Since the ions have a rest mass large compared to that of the $e^{ \pm}$, they will contribute the dominant kinetic energy in the flow, if $N_{1 i}>\left(m_{ \pm} / m_{i}\right)\left(N_{1+}+N_{1-}\right)$. As the plasma encounters the shock, the lighter pairs, which have Larmor radii a factor $m_{ \pm} / m_{i}$ smaller than the ions, form a leading leptonic shock almost identical to the shock found in a symmetric plasma, if $N_{i} \ll\left(N_{+}+N_{-}\right)$. The more massive ions have no chance to respond, since the leptonic shock develops on the lepton cylotron time. They plow on through until they too are magnetically reflected in the enhanced magnetic field of the shock front. An electrostatic field in the direction of flow will be associated with this spatial separation of the ions from the leptons, with electrostatic potential having magnitude $Z e \Phi \sim m_{i} \gamma_{1} c^{2}$. Once the ions are set into gyration by the reflection from the shock front, they too are synchrotron maser unstable, as they gyrate in the much denser background of shock heated pairs.

Simulations of shock flows with ions (Hoshino, Arons, Langdon, Gallant and Max 1990) support these conjectures. In Figure 3, I show the electromagnetic structure from a simulation done in the same manner as the simulations of the purely leptonic shocks, but now containing ions with a charge to mass ratio appropriate to protons, at $36 \omega_{c i}^{-1}$ after the plasma bounced off the simulation wall. In order to keep the computer time finite, the mass ratio is small, $m_{i} / m_{ \pm}=20$, 
and the density ratio is $N_{i} /\left(N_{+}+N_{-}\right)=0.2$, chosen as a compromise to have $N_{i} \ll\left(N_{+}+N_{-}\right)$ but still have most of the kinetic energy density in the upstream flow. In this case, $80 \%$ of the flow energy is in the ions. The upstream flow is almost entirely dominated by the plasma kinetic energy flux $-\sigma=0.005$. While the choice of $\gamma_{1}$ matters only to the relative scale of the flow, in the interests of making the problem be of direct relevance to plerions and pulsars, we chose $\gamma_{1}=10^{6}$, corresponding to protons being accelerated through a few percent of the polar cap
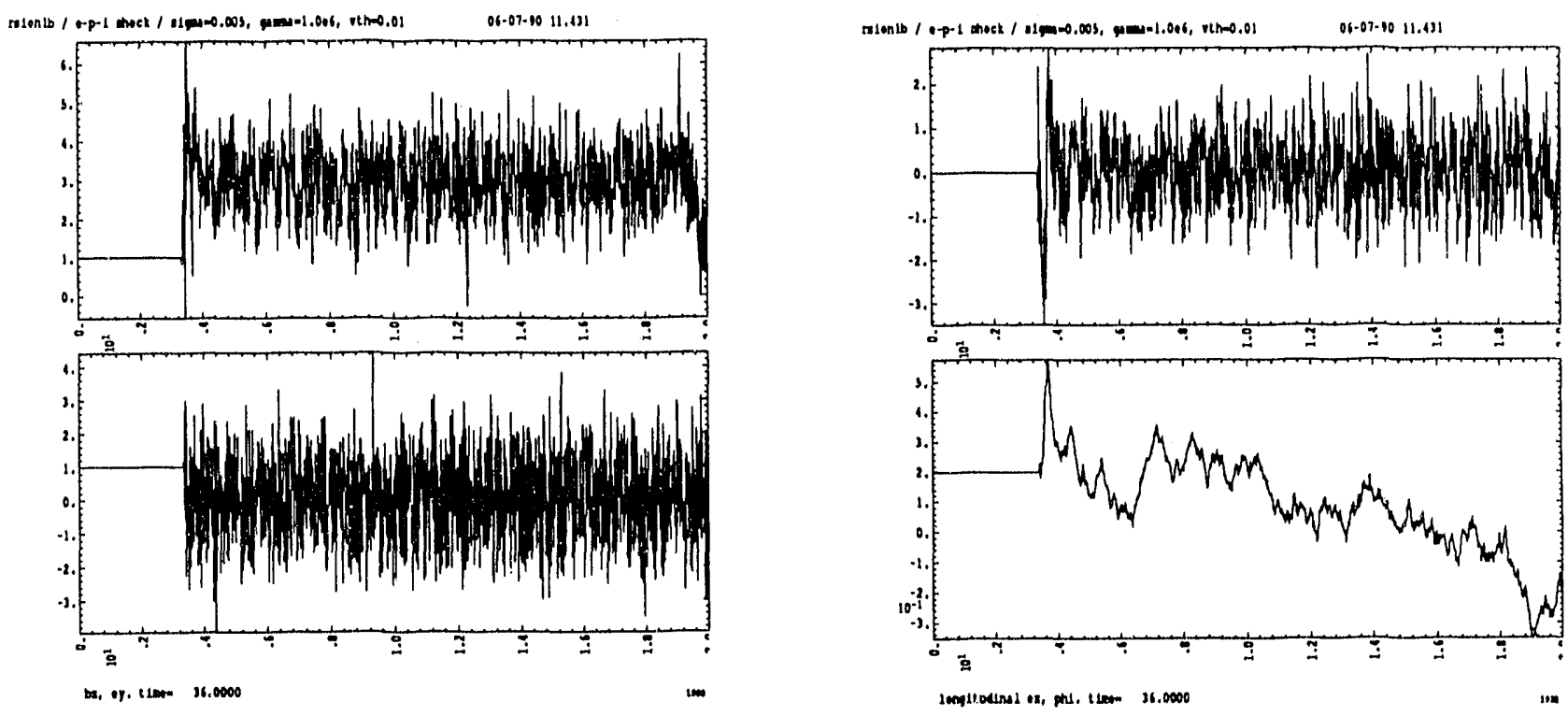

Figure 3: Electromagnetic structure of a relativistic magnetosonic shock in an electron-positronproton plasma, at a time when the shock has crossed $75 \%$ of the simulation grid. Upper left panel: Magnetic field $B_{z}$, with upstream field amplitude equal to unity. Lower left panel: Transverse electric field $E_{y}$, with $E_{y}=\left(v_{1} / c\right) B_{z}$ at the injection wall. Upper right panel: Electrostatic field $E_{x}$. The downstream waves have electrostatic fields with amplitudes roughly one-half the magnitude of the electromagnetic field components, indicating strong elliptical polarization of the post-shock extraordinary modes. Lower right panel: Electrostatic potential in units of $\gamma_{1} m_{i} c^{2} / e$. Since the potential never exceeds 0.6 , the electrostatic field in the shock front acts to decelerate the ion stream, but the reflection is magnetic, as in the shocks in pure pair plasmas.

potential of the Crab pulsar. The presursor does not appear in this simulation, since the grid scale was about equal to $c / \omega_{c \pm}$ based on upstream parameters, which leaves the precursor unresolved. The electrostatic field in Figure 4 shows a strong double layer structure at the shock front, as the ions are first dragged back by the pairs frozen to the field, then gyrate back and pull the pairs forward, while the potential reaches $\sim 0.5 \gamma_{1} m_{i} c^{2} / e$ in the double layer region. Behind the shock, the magnetic field settles down to a mean value of $3 B_{1}$, as one would expect from the MHD jump conditions for such a plasma dominated flow, with intense extraordinary mode noise. In the simulation frame, the average transverse electric field is noise with a mean of zero, while in the shock frame (only approximately well defined, since the shock velocity is not strictly steady 
around its mean value of $c / 3$ ), the mean transverse electric field is approximately constant.

In Figure 4 we show the zoomed views of the $p_{x}-x$ and $p_{y}-x$ projections of ion phase space for a region of the simulation near the shock front itself.
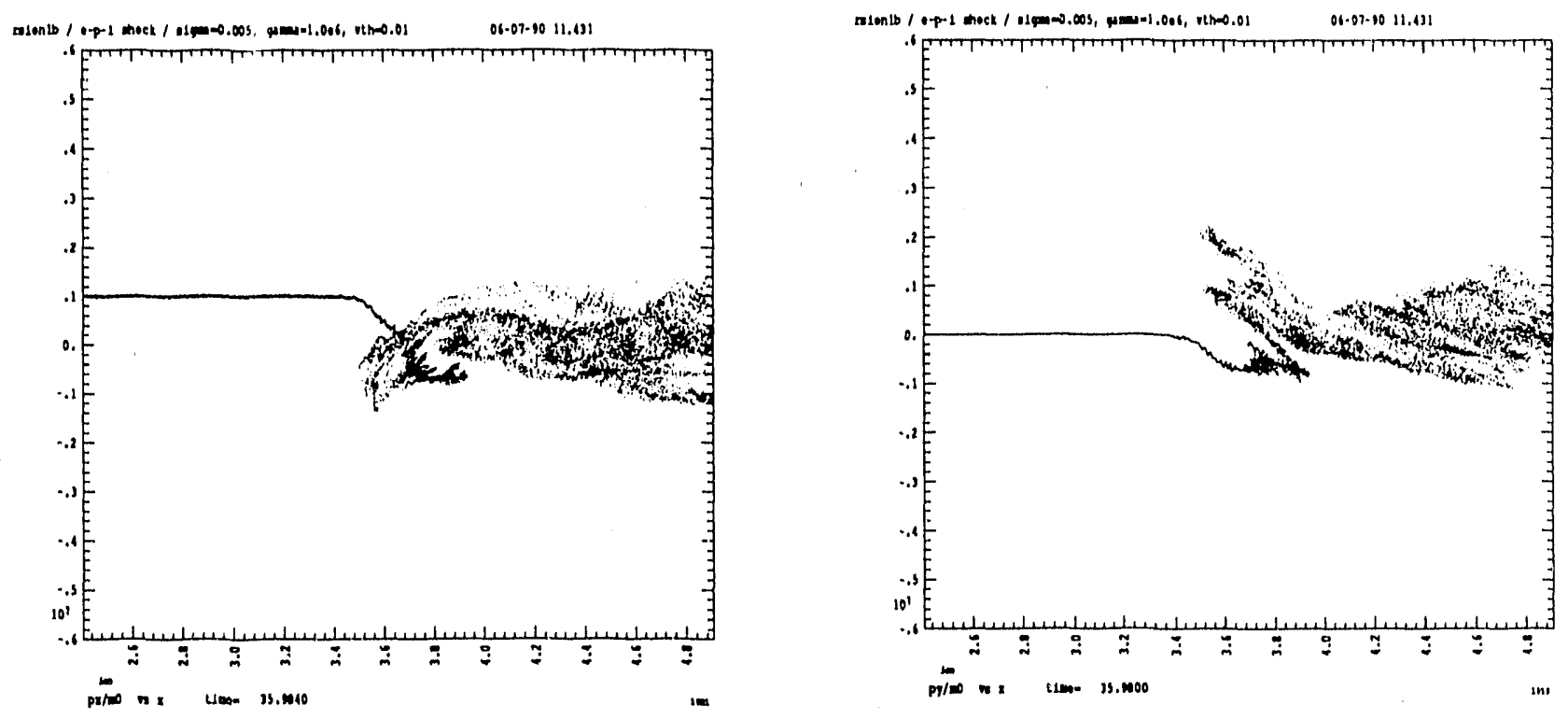

Figure 4: Ion phase space near the shock front. Left panel: Flow momentum versus $x$ for ions near the shock front. Note the initial deceleration of the ion stream in the electrostatic field, followed by approximately two rapidly dissolving loops in the magnetic field. The main body of a Maxwellian distribution is produced within 2-3 Larmor times after the ions begin decelerating in the shock front. Right panel: Transverse momentum versus $x$ near the shock front.

One sees the quasi-coherent reflection of the ions, followed by their rapid thermalization. On this scale, the structure of the lepton shock with its initial thermalization to Maxwellian distributions is instantaneous, achieved as the ions have barely begun to gyrate. In Figure 5, we show the spectra of the downstream electrons and positrons. While the electrons have r ained the Maxwellian distribution found in the symmetric case, the positrons have been transforined, having a power law distribution with $N(E) \propto E^{-3}$ at high energy/particle and energy density comparable to the ions' kinetic energy density in the upstream flow. In other words, a relativistic, low $\sigma$ magnetosonic shock, composed of ions, electrons and positrons, with $N_{i} \ll N_{+}+N_{-}$but with the upstream flow energy dominated by the ions, can accelerate the downstream positrons into a power law distribution with $50 \%$ effiency, with the slope of the power law being very close to what is desired for the macroscopic model. In this particular simulation, the number of particles per grid cell was relatively small (only 10 in the initial state). In other calculations with higher $\sigma$ but with a larger number of particles per cell, and in simulations of the synchrotron maser process in isolation in a uniform medium (Hoshino and Arons 1990), we have found the slope to be closer to 2 . Work is continuing to eliminate this sensitivity to an "experimental" parameter, 
but in any case it is clear that these electron-positron-proton shocks do produce downstream positron spectra whose characteristics are highly desirable for the modeling of plerions.
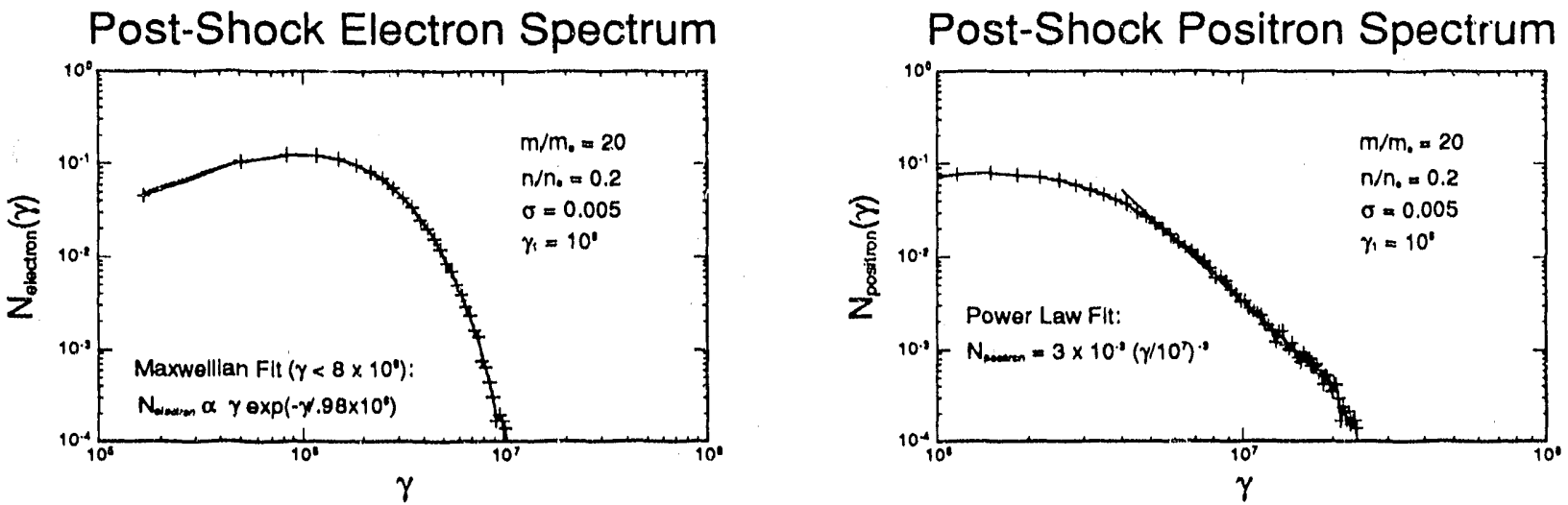

Figure 5: Post-shock lepton distributions, formed from the particles in the region $5<x<18$, with length measured in units of the upstream ion Larmor radius. The shock is located at $x=3.6$, and the right hand wall is located at $x=20$. Left panel: Electrons with the Maxwellian distribution formed at the leading leptonic shock front, unaffected by the presence of the heavy ions. This distribution is the same as the electron and positron distributions formed in a shock with negligible energy flux in heavy ions but with the same value of $\sigma$. Right panel: Positrons, showing the $E^{-3}$ power law distribution at high energy, while th.? spectrum remains quasi-Maxwellian at low energy. The power law extends up to positron energies comparable to $\gamma_{1} m_{i} c^{2}$.

The physics of this acceleration is simple. In this non-symmetric plasma, the extraordinary modes are no longer linearly polarized electromagnetic waves but have a noticeable electrostatic component as they propagate across the magnetic field. For frequencies between the relativistic analog of the lower hybrid frequency and the relativistic cyclotron frequency of the pairs as they emerge from the leading leptonic shock, the extraordinary modes behave like magnetosonic waves in the pair plasma, and have elliptical polarization with the electric vector rotating in the left handed sense with respect to the magnetic field. This is the same sense as the Larmor gyration of the positrons and of the ions. As a result, a positron can linearly absorb the magnetosonic modes at their cyclotron resonance (as well as at higher harmonics) and can gain energy, while the electrons, which have the wrong sense of angular momentum, simply ignore the low frequency ion waves and preserve the temperature gained at the leptonic shock front, $T_{e} \approx \gamma_{1} m_{e} c^{2}$. It is possible to simulate this flow with mass ratios as high as 100 , which gives much larger dynamic range in the particle spectra. Such simulations are in progress.

This mechanism works efficiently only if the collective synchrotron emission of the ions yields extraordinary mode frequencies greater than or equal to the thermal cyclotron frequency of the positrons just behid the leading leptonic subshock. The linear theory shows this is possible only if the upstream thermal momentum dispersion of the ions is small compared to $m_{ \pm} / m_{i}$. This is probably not a severe restriction, both because the acceleration of the ions is likely to be 
by an electromagnetic mechanism (Arons 1983), with the ion energy essentially unique on each streamline, and because adiabatic expansion in the outflow reduces the initial thermal dispersion drastically by the time the shock is reached. To the degree this shock acceleration model works sucessfully, this constraint on the acoustic Mach number of the flow should be regarded as a restriction on any theory of the wind. All of the simulations easily satisfy this requirement.

That this heating process can and should result in power law positron spectra can be shown by an elementary "quasi-linear" argument. If the ion gyration is modeled as a simple homogeneous ring in phase space, while the pairs are modeled as a hot homogeneous plasma, one finds an ion growth rate almost independent of frequency, up to an upper cutoff related to the momentum. dispersion of the ion ring. If the positrons have a power law spectrum $N_{+}(\gamma) \propto \gamma^{-s}$, the linear damping rate at which positrons can cyclotron absorb the magnetosonic modes can be equal to the rate the ions emit the extraordinary modes only if $s=1$. Such detailed balance cannot be achieved at all wave frequencies if the positrons have a Maxwellian distribution. This steady state, "on the spot" argument overdoes the efficiency of the acceleration process - in the "real" world of the simulations, the steady decline of the ions' energy needs to be incorporated in a theory. Nevertheless, the argument does support the appearance of power laws in the positrons as a consequence of the ion maser process. Furthermore, it does suggest that quasi-linear theory is applicable to the post-shock heating process, in spite of the large amplitude of the waves, since the total energy is almost entirely in the particles and even these large amplitude waves can make only moderate disturbances of the particles' orbits.

\section{Application to the Crab Nebula}

Detailed models of astrophysical systems using these results have not yet been constructed, but one can get the flavor of what might happen from the following simple considerations. Suppose the magnetosphere of the Crab pulsar enits a dense flow of pairs, with $\dot{N}_{ \pm} \sim 10^{38} \dot{N}_{ \pm, 38}$ coming out in the broad sector around the rotational equator where the pulsar must emit the wind required to feed the torus of X- and gamma-ray emission in the Nebula - for the nebular geometry and required particle injection rate, see Aschenbach and Brinkmann (1975) and Pelling et al (1987). A number of magnetospheric models, with varying quantitative credibility, suggest such a flow is possible (Arons 1983, Beskin et al 1983). In addition, suppose the magnetospheric electrodynamics causes an outflow of heavy ions to be pulled up from the stellar surface and accelerated to high energy in the outflow around the magnetic equator (Arons 1983 seems to have made the only suggestion of this sort, so far). The particle flux is constrained by the electrodynamics to be close to the Goldreich-Julian flux, $\sim 4 \times 10^{34} Z^{-1} \mathrm{~s}^{-1}$ for this pulsar, where $Z$ is the charge on the ions. The resulting ratio of the mass densities in the wind is $m_{i} N_{i} / m_{ \pm} n_{\text {pair }} \approx 0.7 A / Z \dot{N}_{ \pm, 38}$, where $A$ is the atomic number of the ions. The most likely candidate is fully stripped iron, with $A / Z \approx 2.2$, yielding approximate equipartition between the components of the upstream pair energy. While our simulations so far have been done for somewhat higher ratios of the ion flow energy to that in the pairs, we do not expect major differences for these smaller values.

These ions might be accelerated through a fair fraction of the total polar cap potential $\Phi_{\text {cap }} \sim 10^{17}$ Volts, corresponding to $\gamma_{1} \sim 10^{8}\left(\Phi / \Phi_{c a p}\right)$. The equatorial wind with these 
accelerated ions carries a fraction $\Phi / \Phi_{c a p}$ of the spindown luminosity of the pulsar. If the shock really puts essentially all the energy into the accelerated positrons, as our simulations suggest, and if these positrons radiate essentially all of this in X-rays and gamma-rays, as is indicated by the macroscopic flow models, then one would conclude, from the hard photon luminosity of the nebula, that the ions experience an acceleration potential $\Phi / \Phi_{\text {cap }} \sim 0.1 / I_{45}$, where $I_{45}$ is the moment of inertia of the neutron star in units of $10^{45} \mathrm{cgs}$.

Therefore, our shock acceleration model is consistent with the gross characteristics of what we know about the Crab Nebula. More detailed modeling, including the unusual feature of a Maxwellian component of shock heated electrons in the post shock flow, offers the prospect of seriously constraining models of either the composition of the relativistic outflow from pulsars, or their moments of inertia, or both, as well as providing the obviously useful capability of modeling the particle acceleration characteristics as a function only of the bulk properties of the flow, without having to invoke further free parameters. Therefore, we conclude with the perhaps mildly heretical suggestion (at least relative to the last 10 years of widespread belief) that the outflows from pulsars include both highly accelerated ions and pairs, and that the dominant radiating species in plerionic supernova remnants is the positrons, not the electrons. It has not escaped our attention that such a conclusion has implications for studies of active galactic nuclei also.

We also note that the pairs carry energy comparable to the ions. They are heated in the leading edge of the shock by the subshock in the pairs. During this heating process, they gyrate several times in the rising magnetic field. In so doing, they radiate incoherent synchrotron radiation, which for $\gamma=10^{6}-10^{7}$ occurs in the optical and soft X-ray part of the spectrum, with a luminosity on the order of $10^{35} \mathrm{erg} / \mathrm{s}$. This is just right to explain the "thin wisp" an optical structure located about where the shock should form. Therefore, we suggest the thin wisp is the incoherent emission from the pairs in the shock fronts which we have described here. High resolution study with the space telescope might be interesting.

Our research on relativistic shock waves was supported in part by NSF grant AST-8615816 and by IGPP-LLNL grant 90-14, both to the University of California at Berkeley. It was also supported by NASA astrophysical theory grant NAGW-1301. Part of the work was performed under the auspices of the U.S. Department of Energy at the Lawrence Livermore National Laboratory under contract W-7405-Eng-48.

\section{References}

Alsop, D., and Arons, J. 1988, "Relativistic Magnetosonic Solitons with Reflected Particles in Electron-Positron Plasmas", Phys. Fluids, 31, 839

Arons, J. 1983, "Electron-Positron Pairs in Radio Pulsars"; in Proc. Workshop on ElectronPositron Pairs in Astrophysics, M.L. Burns, A.K. Harding and R. Ramaty, eds. (New York: American Institute of Physics), 163

Hoshino, M. and Gallant, Y.A. 1990, "Synchrotron Instability, Absorption and Suprathermal Particle Acceleration in Relativistic, Magnetosonic Shock Waves", to be submitted to Ap.J. 
Aschenbach, B., and Brinkmann, W, 1975, "A Model of the X-Ray Structure of the Crab Nebula", Astron. and Ap., 41, 147

Begelman, M.C., and Kirk, J. 1990, "Shock Drift Acceleration in Superluminal Shocks: A Model for Hotspots in Extragalactic Radio Sources", Ap.J., in press

B.ll, A.R. 1978, "The Acceleration of Cosmic Rays in Shock Fronts", Mon. Not. Roy. Astron. Soc., 182,147

Beskin, V.S., Gurevich, A.V., and Istomin, Ya. N. 1983, "Electrodynamics of Pulsar Magnetospheres", Zh. Eksp. Teor. Fiz., 85, 401 (Soviet Physics - JETP, 58, 235)

Birdsall, C., and Langdon, A.B. 1985, Plasma Physics via Computer Simulation (New York: McGraw-Hill)

Chiueh, T. 1989, "Relativistic Solitons and Shocks in Magnetized $e^{-}-e^{+}-p^{+}$Fluids", Phys. Rev. Lett., 63, 113

Ellison, D.C., Jones, F.C., and Reynolds, S.P. 1990, "First-Order Fermi Particle Acceleration by Relativistic Shosks", $A_{k} I_{\text {., }}$ in press

Emmering, R.T, and Chevalier, R.A. 1987, "Shocked Relativistic Magnetohydrodynamic Flows with Application to Pulsar Winds", Ap. J., 321, 334

Gallant, Y.A., Hoshino, M., Langdon, A.B., Arons, J., and Max, C.E. 1990, "Structure of Relativistic Magnetosonic Shock Waves in Electron-Positron Plasmas", to be submitted to Ap. $J$.

Gold, T. 1969, "Rotating Neutron Stars and the Nature of Pulsars", Nature, 221, 25

Hoshino, M., and Arons, J. 1990, "Differential Heating and Acceleration of Positrons by Synchrotron Maser Instabilities", submitted to Pinys. Fluids

Arons, J., Langdon, A.B., Gallant, Y.A., and Max, C.E. 1990, "Differential Heating and Acceleration of Positrons in Electron-Positron-Proton Relativistic Magnetosonic Shocks", to be submitted to $A p$. $J$.

Kennel, C.F., and Pellat, R. 1976, "Relativistic Nonlinear Waves in a Magnetic Field", J. Plasma Phys., 15, 335

Kennel, C.F., and Coroniti, F.V. 1984a, "Confinement of the Crab Pulsar's Wind by its Supernova Kemnane", Ap. J., 283, 694.

1984b, "Magnetohydrodynamic Model of Crab Nebula Radiation", ibid., 710

Kundt, W., and Krotscheck, E. 1980, "The Crab Nebula - a Model", Astron. and Ap., 83, 1

Langdon, A.B., and Lasinsli, B.F. 1976, "Electromagnetic and Relativistic Plasma Simulation Models", in Methods in Computational Physics, 16, B. Alder, S. Fernbach and M. Rotenberg, eds. (New York: Academic Press), 327

Arons, J., and Max, C.E. 1988, "Structure of Relativistic Magnetosonic Shocks in Electron-Positron Plasmas", Phys. Rev. Lett., 61, 779

Michel, F.C., Scowen, P.A., Dufour, R.J., and Hester, J.J. 1990, "Observations of a Pulsar Wind: CCD Polarimetry of the Crab Nebula", ApJ., in press

Pacini, F. 1967, "Energy Emission from a Neutron Star", Nature, 216, 567 
Pelling, R.M., Paciesas, W.S., Peterson, L.E., Makashima, K., Oda, M., Ogawara, Y., and Miyamoto, S. 1987, "A Scanning Modulation Collimator Observation of the High Energy X-Ray Source in the Crab Nebula", Ap. J., 319, 416

Piddington, J.H. 1957, "The Crab Nebula and the Origin of Interstellar Magnetic Fields", Aust. J. Phys., 10, 530

Rees, M.J., and Gunn, J.E. 1974, "The Origin of the Magnetic Field and Relativistic Particles in the Crab Nebula", Mon. Not. Roy. Astron. Soc., 167, 1

Yoon, P. 1990, "Amplification of a High-Frequency Electromagnetic Wave by a Relativistic Plasma", Phys. Fluids, 2B, 867

Zheleznyakov, V.V., and Suvorov, E.V. 1972, "Results and Problems in the Investigation of the Synchrotron Instability", Ap. and Space Sci., 15, 24 

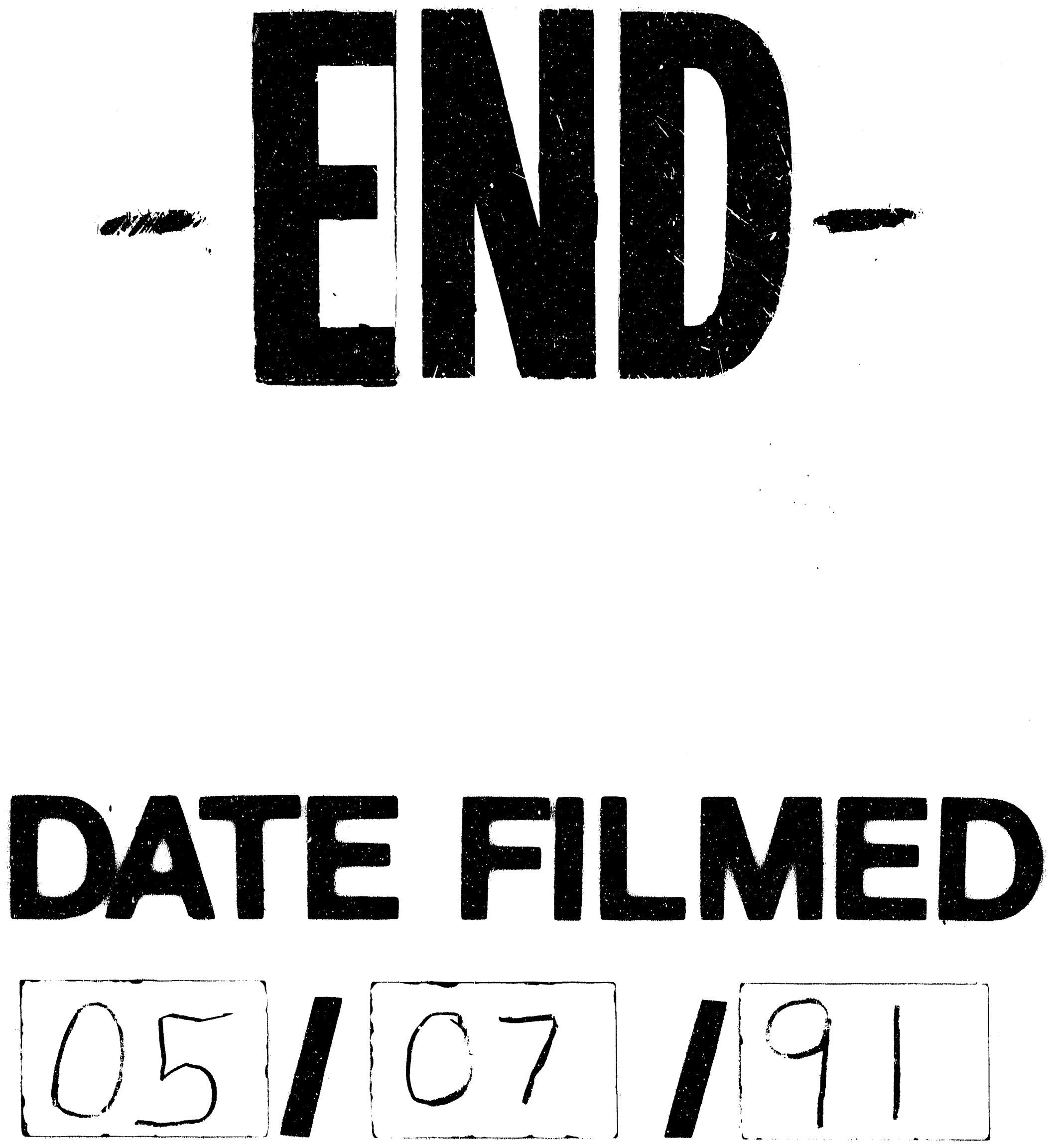
